\title{
Parameter estimation under non-stationary circumstances using extended signal model
}

\author{
M. Lechtenberg ${ }^{1}$, J. Götze ${ }^{1}$, K. Görner ${ }^{2}$, and C. Rehtanz ${ }^{2}$ \\ ${ }^{1}$ Information Processing Lab, TU Dortmund, Otto-Hahn-Str. 4, 44227 Dortmund, Germany \\ ${ }^{2}$ Institute of Energy Systems, Energy Efficiency and Energy Economics, TU Dortmund, Emil-Figge-Str. 70, 44227 Dortmund, \\ Germany
}

Correspondence to: M. Lechtenberg (matthias.lechtenberg@tu-dortmund.de)

Received: 4 December 2013 - Accepted: 27 August 2014 - Published: 10 November 2014

\begin{abstract}
In the context of transient processes in power system measurements, a sampled signal is analyzed with respect to electro-magnetic influences. These are most likely superposed sinusoids that can be found next to the fundamental system sinusoid. However, such signal is not stationary, i.e. has a varying model order which means that temporary and exponentially damped sinusoids appear eventually. In our previous work, the exponential damping was only considered indirectly but not explicitly incorporated in the signal model used. Regarding the amplitude estimation, this led to inaccuracies since the amplitude was incorrectly assumed constant within a window of samples. In this paper, we use ESPRIT's ability to also estimate a parameter of exponential damping, improve the corresponding signal model, advance the amplitude estimation algorithm and back projection algorithm. Especially short-term signal components can be tracked far more precise.
\end{abstract}

\section{Introduction}

Modern power system operation requires sophisticated monitoring and analysis since the power distribution policy changes from unidirectional central power generation to a highly meshed grid of distributed and varying generation. PMUs are widely recognized for their accurate synchrophasor measurement subject to dynamic conditions. However, only stationary signals and modulated signals with low frequency components (i.e. mainly electro-mechanic transients) are considered, complying with the requirements of the IEEE Standard for synchro-phasors C37.118-2011; reviewed in this context by Görner et al., 2013). Electro- magnetic transients e.g. induced by switchings in the network are not considered.

When estimating these electro-magnetic signal components, it has to be considered that these components are not part of the signal for all time. They are induced by events like switchings or line faults and fade out exponentially. In terms of a model, the net can be seen as a circuit of inductances, capacitances and resistances forming meshes with individual resonance frequencies. Such mesh might be excited by an event and (in consequence) may start to oscillate. This oscillation is typically exponentially damped. The mentioned events become more frequent these days due to the evolution towards more dynamic operation of the net.

Waveform analysis is a central part of this monitoring and analysis process. Yet, waveform analysis can only be done using sampling windows (Banerjee and Srivastava, 2012). Focusing on the prior to this work not explicitly considered exponential damping, it is worth mentioning that subspace analysis and frequency estimation are not affected by the damping influencing the current window. Furthermore, the subspace-based frequency estimation algorithm ESPRIT can also estimate the parameter of exponential damping. However, the fact that a sample at the beginning of a sampling window is affected by exponential damping differently than the on at the end of the window has drawbacks on the amplitude estimation, naturally. This can be circumvented by incorporating the exponential fading parameter explicitly.

As a framework for this paper, the waveform analysis is done by subspace extraction in order to enable the detection and estimation of electro-magnetic parameters aside the system's fundamental frequency. The subspace-based parameter estimation algorithm ESPRIT (Paulraj et al., 1986) is 


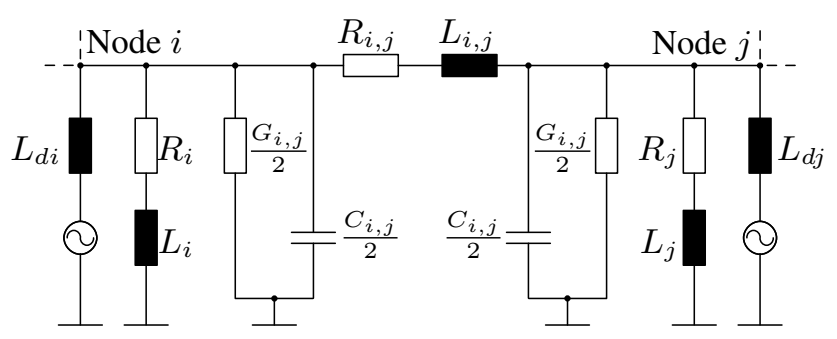

Figure 1. PI-modeled transmission line between nodes $i$ and $j$.

supported by a subspace tracker of the PAST-family (Yang, 1995a). Further (complex) amplitude estimation is done with the help of a Least-Squares (Sorenson, 1970) approach. The extracted parameters are gathered and rated by the DaPT algorithm (Lechtenberg and Götze, 2010; Lechtenberg et al., 2012). The extension back projection introduced in Lechtenberg et al. (2013) will also be used to emphasize the benefit.

In the following subsection, the signal model with and without exponential damping will be explained. The framework including ESPRIT, PAST, DaPT, Back Projection and LS-based amplitude estimation are reviewed in Sect. 2. The paragraphs explaining LS-based amplitude estimation also introduce signal reconstruction and RMSE calculation for evaluation. A simulation demonstrates the application of these methods in Sect. 3. This paper is completed by a summary of the work and the results in a final section, also providing a glance at future work.

\subsection{Line modeling and signal model}

The transmission net can be modeled as a circuit of resistances, inductances and capacitances with partly overlaid meshes, see Fig. 1. Each mesh with inductances and capacitances taken from the circuit has its own resonance frequency and can be excited by a switching event. In such case, each participating mesh in a node provides its resonance frequency as a superposed sinusoid in the voltage/current signal of this node.

These sinusoids appear during a transient process when the transmission net merges from one steady-state condition into another. A resulting signal model for one segment of constant model order might be written as follows:

$x(n)=\sum_{i=1}^{p} c_{i}(n) e^{j n \omega_{i} f_{\mathrm{s}}^{-1}}+w_{\mathrm{awgn}}(n)$

However, this signal model does not consider exponential damping. In consequence, such effect would indirectly be part of the complex amplitude $c_{i}(n)$; this indicates that the model is not sufficient. The following equation adds the missing exponential factor:

$x(n)=\sum_{i=1}^{p} c_{i}(n) e^{-\gamma n f_{\mathrm{s}}^{-1}} e^{j n \omega_{i} f_{\mathrm{s}}^{-1}}+w_{\mathrm{awgn}}(n)$
The input samples $x(n)$ may also be used in the form of a vector $\boldsymbol{x}(n)$ describing a set of subsequent samples. The number of sinusoids (rank) is $p$. The complex amplitude $c_{i}(n)=a_{i}(n) \exp \left(j \varphi_{i}\right)$ is built of the (initial) amplitude $a_{i}(n)$ and the phase $\varphi_{i}$ (at time instant $n=0$ ). $\omega_{i}=2 \pi f_{i}$ describes the angular frequency. The parameter $\gamma$ is the exponential fading factor; since the argument is negative, a positive $\gamma$ indicates damping. The component $\left\{c_{1}, \omega_{1}\right\}$ represents the fundamental frequency. The resonance frequency of an excited LC-oscillation mesh is embodied in $\left.\omega_{i}\right|_{i>1}$. Note that to increase comprehensibility, the signal model is reduced to an only-exponential model although real-valued measurements would require a description with sines or cosines (or - via Euler's identity - doubled exponentials).

\section{Process chain}

\subsection{Subspace estimation}

The concept of the presented parameter estimation was already discussed before (e.g. Lechtenberg and Götze, 2011). The samples from the measurement are prepared with a sliding window before the respective data is fed to the subspace estimator.

The samples' space can be described by a set of vectors forming a basis. The obvious way to do this is to use windowed sample data to estimate the auto-correlation matrix (e.g. via exponentially weighted averaging) and to perform an eigenvalue/-vector decomposition of this matrix. These eigenvectors form one possible basis of the samples' space. Knowing the rank $p$, the signal's subspace can be extracted by selecting the vectors corresponding to the $p$ greatest eigenvalues. Consequently, the other vectors span the noise subspace.

A less computational complex method to find a basis for the signal's subspace is depicted by the so-called subspace trackers. They do not produce eigenvectors; they provide vectors forming a basis describing the same space as a basis defined by eigenvectors does. Next to algorithms like PROTEUS (Champagne and Liu, 1998) or YAST (Badeau et al., 2008) the class of PAST-based algorithms (Yang, 1995b) is common in such context. In this work, the OPAST algorithm (Abed-Meraim et al., 2000) was identified to be a good choice.

PAST-based algorithms are based on the nearly unconstrained minimization of the cost function

$J(\hat{\mathbf{W}})=E\left\|\boldsymbol{x}-\hat{\mathbf{W}} \hat{\mathbf{W}}^{H} \boldsymbol{x}\right\|^{2}$

where $\hat{\mathbf{W}}$ is not constrained to hold eigenvectors. The minimization is based on the idea of gradient-descent methods and incorporates exponential weighting for updating $\hat{\mathbf{W}}$ which increasingly better approximates the basis vectors $\mathbf{W}$ (Yang, 1995b). In exponential weighting $b(n)=\beta b(n-1)+$ 
$(1-\beta) \hat{b}$, the factor $\beta$ is also called forgetting factor. The pseudo window length $w_{\mathrm{PAST}}=\frac{1}{1-\beta}$ gives an impression on the rage of samples affecting the current average.

Assuming valid results of OPAST, the following equations provide noise samples $\boldsymbol{w}$ and an estimation of the noise power $\sigma^{2}$ :

$\boldsymbol{w}(n)=\boldsymbol{x}(n)-\mathbf{W} \boldsymbol{y}$

$\hat{\sigma}_{\text {awgn }}^{2}=\|\boldsymbol{w}(n)\|_{2}^{2}$

with an internal PAST variable $\boldsymbol{y}$ only weakly related to eigenvalues.

\subsection{Parameter estimation}

The a priori selected basis vectors describing the desired signal are fed to the subspace-based parameter estimator ESPRIT (Paulraj et al., 1986). It is based on the analysis of the rotational invariance of two subsequent basis vectors. Due to the principle of rotational invariance, these vectors should only differ in a constant rotation $\left.\exp \left(j \cdot n \cdot \Omega_{i}\right)\right|_{n=1}$ (with $\Omega_{i}=2 \pi \frac{f_{i}}{f_{\mathrm{s}}}$ ) depending on the eigenfrequency $f_{i}$, which is the desired parameter.

Remembering the signal model in Eq. (2), the signal can also be described by subspaces like in the equation

$$
\begin{aligned}
E\left[\boldsymbol{x}^{H}\right] & =\mathbf{S}(n)+\sigma_{\text {awgn }}^{2} \mathbf{I} \\
\text { with } \boldsymbol{x} & =\mathbf{A}(n, \boldsymbol{\omega}) \boldsymbol{c}(n)+\boldsymbol{w}_{\text {awgn }}(n) .
\end{aligned}
$$

The matrix A contains the exponential functions $\exp \left(j \omega_{i} \frac{n}{f_{\mathrm{s}}}\right)$ (and $\exp \left(-\frac{\gamma_{i}}{f_{\mathrm{s}}}\right)$ ) to form the superposition in the horizontal dimension and the burst description in the vertical dimension. $\mathbf{S}(n)$ describes the signal subspace, which can be written as a product of the eigenvectors $\mathbf{W}(n)$ and the eigenvalues (on a diagonal matrix) $\mathbf{D}(n)$ :

$\mathbf{S}(n)=\mathbf{W}(n) \mathbf{D}(n) \mathbf{W}(n)^{H}$.

One time-step can be described by a multiplication with a diagonal matrix $\boldsymbol{\Phi}$ having diagonal entries $\exp \left(2 \pi j \cdot \frac{f_{i}}{f_{\mathrm{s}}}-\frac{\gamma_{i}}{f_{\mathrm{s}}}\right)$ :

$\mathbf{W}(n+1)=\mathbf{W}(n) \boldsymbol{\Phi}$.

The key idea of ESPRIT is to estimate the rotation $\boldsymbol{\Phi}$. Thinking of the burst dimension of $\mathbf{A}$, this rotation is also the factor between the upper $N-1$ lines of $\mathbf{W}$ and the lower. Due to noise, $\hat{\boldsymbol{\Phi}}$ will have off-diagonal elements. So an EVD is performed on $\hat{\boldsymbol{\Phi}}$. The frequencies can be extracted from these eigenvalues by calculating their angle. These frequencies are the input of DaPT.

Recapitulating the idea of ESPRIT, the to be estimated factor is an exponential function with a complex argument. However, this algorithm also works for factors like

$\phi_{i, i}=e^{\left(j \cdot \frac{\omega_{i}}{f_{\mathrm{s}}}-\frac{\gamma_{i}}{f_{\mathrm{s}}}\right)}$.

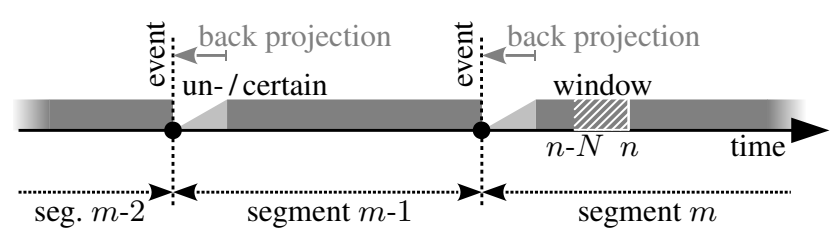

Figure 2. Quasi-stationary segments of measurement samples, uncertainty regions, sample window and back projection concept.

As can be seen, the idea of ESPRIT is not restricted to rotational poles but allows poles aside the unit circle (Badeau et al., 2003). The exponential factor can be retrieved by calculating the logarithm of the absolute value of a $\phi_{i, i}$.

\subsection{Database-assisted Parameter estimation - DaPT}

Assuming the feed of ESPRIT to contain a few more basis vectors than the rank of the signal, these additional vectors belong to the noise subspace and are named auxiliaries (Yang, 1995b). In contrast to the signal's vectors, theses will not result in constant parameter estimations within the ESPRIT algorithm. The idea of DaPT (Database-assisted Parameter estimation, Lechtenberg and Götze, 2011) is to rate the temporal presence of each frequency.

Entries with high rating can be hypothesized as part of the desired signal and entries with a low rating are assumedly noise and to be forgotten. By this, the algorithm recognizes a change in rank by simply counting entries with high ratings. This rank is advanced by a small number (the auxiliaries) and looped back to the block that selects the vectors to be fed to ESPRIT.

In every recursion, the incoming frequency estimation is mapped to the most suitable database entry. Ideally, both values match neglecting the measurement noise. For entries that have been successfully mapped, the quality is increased (up to a maximum value); for others, it is decreased (down to zero). A zero-quality-entry will be deleted. The frequency entry is corrected according to the drift measures' severity indexes exceeding a threshold. The measures' values are exponentially weighted over time (double-exponential smoothing) and its severity index is updated sign-depended.

This database has entries for each signal component containing frequency $f_{i}$, fading parameter $\gamma_{i}$ and (complex) amplitude $c_{i}$. The complex amplitude is to be estimated separately (see Sect. 2.5). Amongst other fields, DaPT also has a field valuing the quality of an entry, which is the actual rating. It rates how often a frequency has been part of the last estimation recursions. Based on that, components can be marked reliable (i.e. present), and therefore are accounted for rank estimation and output. 


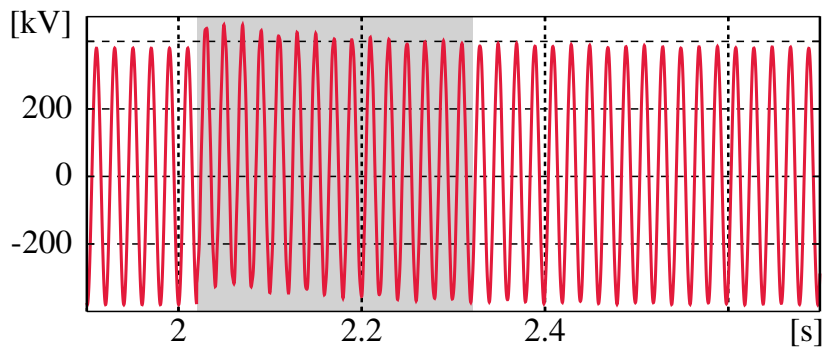

Figure 3. Input signal with an event shortly after $2 \mathrm{~s}$ producing decaying DC offset and additional sinusoids.

\subsection{Segmentation and back projection}

Anticipating, after the estimation of all signal parameters, a reconstruction of the signal is possible. The difference of this reconstruction to the input signal is an indicator for the fulfillment of the assumptions necessary for the algorithms. Calculating the MSE of this signal error and monitoring (by statistical means like $n \cdot \sigma$-rules) enables the robust detection of a violated stationarity assumption. These values can be expected to be fairly constant and may not contain significant steps. If a significant violation is detected, the time instant indicating a transient process from one stationary state to another may be found. This evaluation provides a so-called segmentation of the signal (Lechtenberg et al., 2013).

Furthermore, with the help of this segmentation information, it is possible to reconstruct the parameters without the uncertainties of the compromised windows. When an event is detected and the segmentation is triggered, a timer is set to wait a short while until the parameters can be expected to be valid/certain again (see Fig. 2). With the help of these valid parameters, the estimation for the time between the event and the time-out can be redone time-reversed. Of course, this requires the input samples to be memorized back to the time instant of segmentation.

Although this enables the reparation of the corrupted estimates, the delay resulting from the sliding window principle cannot be compensated, obviously. In consequence, the resulting parameter estimation provides clean estimates both prior to (causality principle) and after (back projection) the event.

\subsection{Estimation of complex amplitude}

Phase and amplitude estimation can be obtained by a simple LS approach referring to the signal model in Eq. (6). Since time index, frequency and exponential factor are known, the matrix A can be built. Together with the samples, the complex amplitude can be estimated. Using the same method, the synchrophasor can be determined by referencing a constant frequency - i.e. the system's fundamental frequency - and calibrating an offset-phase to adapt to the UTC-time (Martin et al., 2006). However, a real-time system like FPGA is rec-

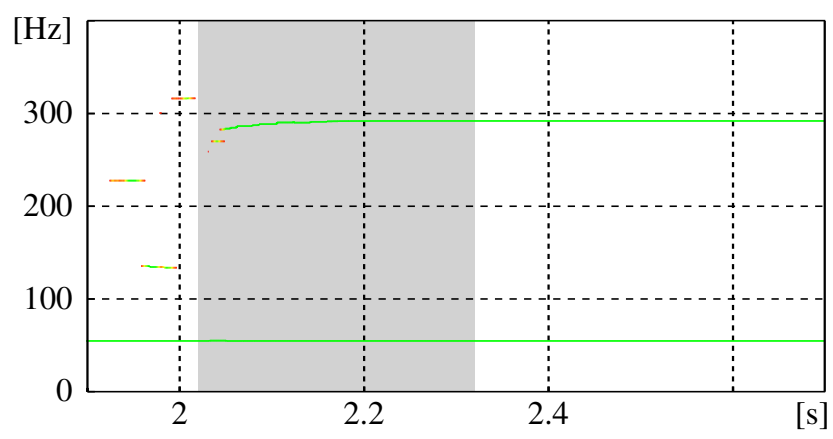

Figure 4. Estimation of frequency parameter without back projection.

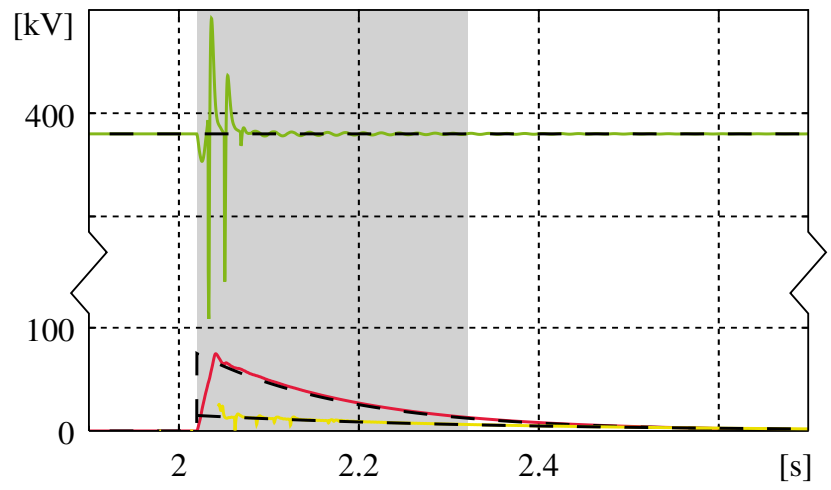

Figure 5. Amplitude estimation without back projection using signal model without damping (dashed: theoretical; with a break in scale).

ommended for meeting the temporal requirements of PMU measurements.

Since the construction of the matrix $\mathbf{A}$ is not constrained to a fixed window length and window time offset but only needs to fulfill the LS requirement, i.e. an overdetermined system of equations, the application of back projection (Sect. 2.4) is straight forward.

The signal model and the matrix A, resp., can easily be used to reconstruct the input signal. This enables the calculation of the (R)MSE ((rooted) mean squared error) between this reconstruction and the original input samples:

$\operatorname{RMSE}(n)=\sqrt{\frac{1}{N} \sum_{i=n-N+1}^{n}[x(i)-\mathbf{A}(i, \boldsymbol{\omega}) \boldsymbol{c}(i)]^{2}}$.

\section{Simulation}

The signal processing is done with The Mathworks MATLAB $^{\text {TM }}$. The sampling frequency is $f_{\mathrm{s}}=5 \mathrm{kHz}$; the window length is $w_{1}=100$ and the PAST forgetting factor is $\beta=0.8$. The simulation is based on an electric power signal of $50 \mathrm{~Hz}$ powered to $380 \mathrm{kV}$ that is constantly present. At the time of $2 \mathrm{~s}$, two other signal components are added: 


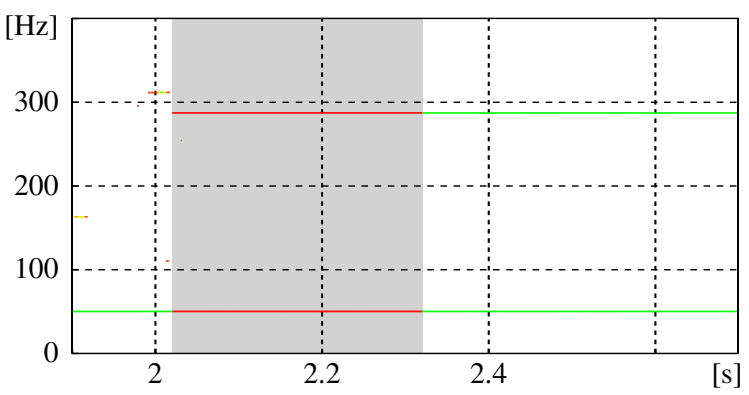

Figure 6. Estimation of frequency parameter with back projection.

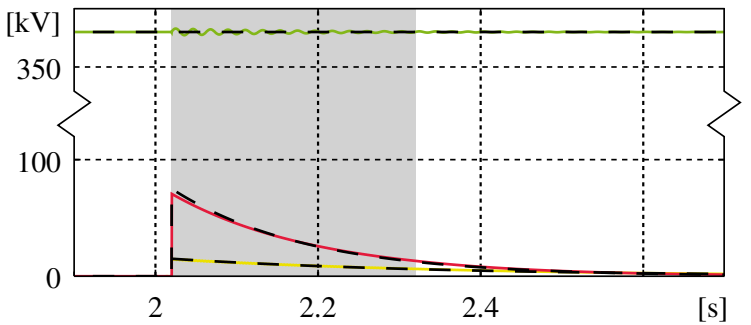

Figure 7. Amplitude estimation without back projection (dashed: theoretical; with a break in scale.)

another sinusoid of $\approx 290 \mathrm{~Hz}$ at $\approx 15 \mathrm{kV}$ with an exponential damping factor of 3 and a direct component of $\approx 75 \mathrm{kV}$ with an exponential factor of 6 . The signal has additive white Gaussian noise resulting in an SNR of $80 \mathrm{~dB}$. The following demonstration is based on one run of this simulation producing a signal like in Fig. 3.

When parameter estimation is done without respecting the constraint of stationarity, the results will be corrupted right after the model order changes. The less samples prior the model order change affect the estimation (due to exponential averaging) the less corrupted the estimates are. This is illustrated by Fig. 4 for frequency estimation and Fig. 5 for subsequent amplitude estimation.

In Lechtenberg et al. (2013), the concept of (segmentation and) back projection is introduced. Using back projection circumvents the corruption by recalculating the threatened region of samples. Figure 6 demonstrates this for the frequency estimation. However, in contrast to the frequency being correctly modeled as constant, the amplitude is also modeled as constant; this is not correct due to the not considered exponential damping, see Eq. (1). In Fig. 7, the estimates are clearly off the true values.

Using the signal model in Eq. (2) for the amplitude estimation requires more information; it utilizes a set of input samples and the ESPRIT-estimated parameters $f_{i}$ and $\gamma_{i}$ for frequency (Fig. 6) and damping (Fig. 8) to solve a set of equations for the only missing parameter $c_{i}$. As Fig. 9 visualizes, the signal model supports the complete signal. A comparison of the RMSE evaluating the estimation against the input sig-

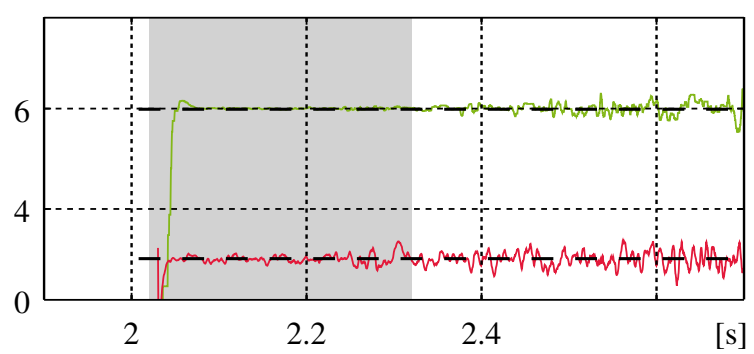

Figure 8. Estimation of exponential damping factor by ESPRIT (dashed: theoretical).

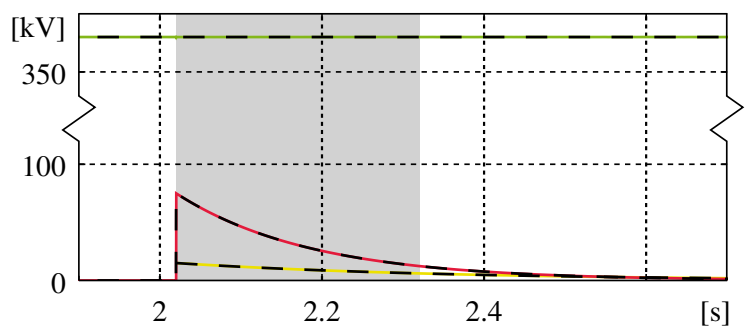

Figure 9. Fully enabled amplitude estimation (dashed: theoretical; with a break in scale).

nal also confirms this study contrasting both prior presented simulations (Fig. 10).

Although the amplitudes of an estimation without support for damping inside the signal model will not match (see Fig. 7), these amplitudes can be used to estimate the damping factor $\gamma_{i}$ as can be seen in Fig. 11. This estimation of $\gamma_{i}$ is less corrupted than in Fig. 8 since the effect of back projection simulates stationarity. The segmentation information from the back projection and the information about the exponential damping can jointly be used to estimate the initial amplitude of such temporary sinusoid; Fig. 12 demonstrates this.

\section{Conclusions}

Parameter estimation in an environment of superposed sinusoids in which not all of them are continuously present but emerging step-like and fading in an exponentially damped fashion is not trivial. There are three main challenges:

1. the estimation of the number of currently present sinusoids (estimation of rank/model order),

2. the segmentation of the signal at the time instants of model order change (to ensure stationarity), and

3. the choice of the correct signal model.

Only if all of these items are considered, it is possible to extract all signal parameters (i.e. frequency, exponential 


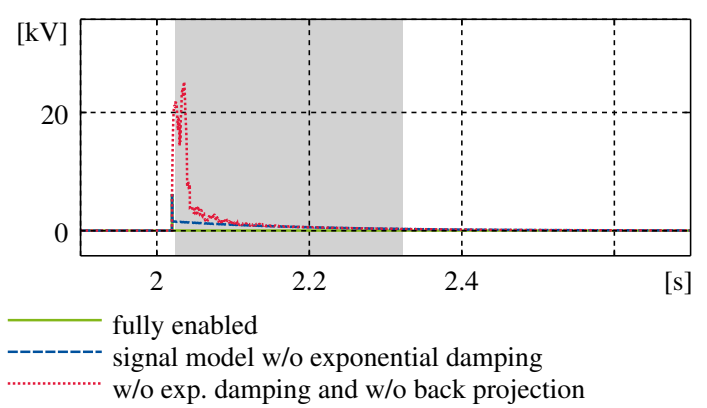

Figure 10. RMS of difference between reconstructed signal and input signal, RMSE.

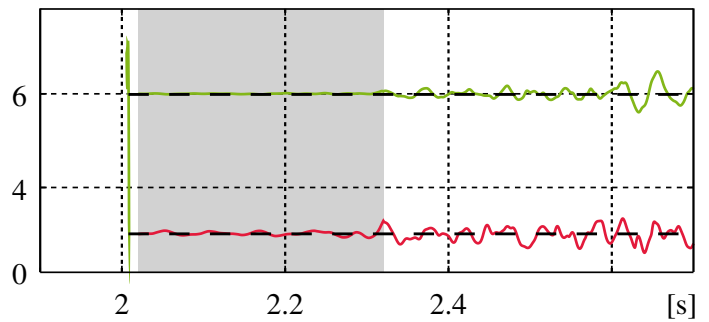

Figure 11. Estimation of exponential damping factor by logarithm of estimated amplitude (dashed: theoretical).

damping factor, (initial) amplitude, and segmentation information). The success may be evaluated by reconstructing the signal and comparing it to the input sequence.

In this paper, we advanced the signal model of superposed sinusoids by adding an exponential factor for damping. By exploiting ESPRIT's capability of not only estimating the frequency parameter but the complete complex pole, the subsequent least-squares estimation can match the signal model with the only on free parameter; the complex amplitude describes the phase offset and the initial amplitude of a sinusoid.

Considering the direct component to be a sinusoid of $0 \mathrm{~Hz}$, it can jointly be estimated with the other signal components. In terms of complex computation, the only difference is that real sinusoids have to be considered with a positive and a negative frequency (Euler's identity) but the direct component only with one.

Future plug-ins to this work may use information from all phases of the 3-phase transmission system. Amplitude modulations (which can also be described as sinusoids very close in frequency) are still a challenge to be handled. A possible FPGA implementation may be considered for the fully enabled system.

Acknowledgements. This work was supported by $\mathbf{D F G}$ (German Research Foundation).

Edited by: W. Mathis

Reviewed by: two anonymous referees

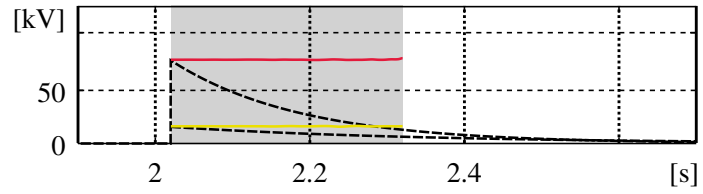

Figure 12. Estimation of initial amplitude and (dashed) theoretical amplitude.

\section{References}

Abed-Meraim, K., Chkeif, A., and Hua, Y.: Fast orthonormal PAST algorithm, IEEE Signal Proc. Lett., 7, 60-62, 2000.

Badeau, R., Richard, G., and David, B.: Adaptive ESPRIT algorithm based on the PAST subspace tracker, Int. Conf. Acoust. Spee., Vol. 6, 229-232, 2003.

Badeau, R., Richard, G., and David, B.: Fast and Stable YAST Algorithm for Principal and Minor Subspace Tracking, IEEE T. Signal Proces., 56, 3437-3446, 2008.

Banerjee, P. and Srivastava, S.: A Subspace-Based Dynamic Phasor Estimator for Synchrophasor Application, IEEE T. Instrum. Meas., 61, 2436-2445, 2012.

Champagne, B. and Liu, Q.-G.: Plane rotation-based EVD updating schemes for efficient subspace tracking, IEEE T. Signal Proces., 46, 1886-1900, 1998.

Görner, K., Lechtenberg, M., Rehtanz, C., and Götze, J.: A Method for Adaptive Time-Synchronized Measurement during Transients, in: 2013 International Conference on Power Systems Transient, 10th, 2013.

Lechtenberg, M. and Götze, J.: A Database Post-Processing Approach for Robust Frequency Estimation, in: Kleinheubacher Tagung, 2010.

Lechtenberg, M. and Götze, J.: Database Assisted Frequency Estimation, in: 4th IEEE International Conference on Computer Science and Information Technology, 2011.

Lechtenberg, M., Götze, J., Rehtanz, C., and Görner, K.: Database Assisted Frequency Estimation for Power System Measurement, in: 2012 IEEE Electrical Power and Energy Conference, 2012.

Lechtenberg, M., Götze, J., Görner, K., and Rehtanz, C.: Improved Parameter Estimation under Non-Stationary Circumstances using Segmentation and Back Projection, in: IEEE International Conference on Smart Instrumentation, Measurement and Applications (ICSIMA 2013), Kuala Lumpur, Malaysia, 2013.

Martin, K., Benmouyal, G., Adamiak, M., Begovic, M., Burnett, R. J., Carr, K., Cobb, A., Kusters, J., Horowitz, S., Jensen, G., Michel, G., Murphy, R., Phadke, A., Sachdev, M., and Thorp, J.: IEEE Standard for Synchrophasors for Power Systems, IEEE Std C37.118-2005 (Revision of IEEE Std 1344-1995), 1, 1-57, 2006.

Paulraj, A., Roy, R., and Kailath, T.: A subspace rotation approach to signal parameter estimation, P. IEEE, 74, 1044-1046, 1986.

Sorenson, H. W.: Least-squares estimation: from Gauss to Kalman, IEEE Spectrum, 7, 63-68, 1970.

Yang, B.: An extension of the PASTd algorithm to both rank and subspace tracking, IEEE Signal Proces. Lett., 2, 179-182, 1995a.

Yang, B.: Projection approximation subspace tracking, IEEE T. Signal Proces., 43, 95-107, 1995b. 